\title{
Colloid Science
}

\section{Flow curves of an adsorbed protein layer at the saliva-air interface}

\author{
H. J. Holterman, E. J. 's-Gravenmade ${ }^{1}$ ), H. A. Waterman, J. Mellema, and C. Blom \\ Rheology Group, Faculty of Applied Physics, University of Twente, Enschede, The Netherlands \\ 1) Laboratory for Materia Technica, University of Groningen, Groningen, The Netherlands
}

\begin{abstract}
At the air-liquid interface of human saliva a protein layer is adsorbed. An apparatus is described with which a flow curve of this layer was measured. In the majority of samples the viscosity of the surface layer changed gradually and could be described by a power-law dependence on the shear rate. The zero-shear viscosity was 1-100 MPa.s. In some saliva samples a sharp yield point was observed.
\end{abstract}

Key words: Saliva, human; rheology; protein adsorption.

\section{Introduction}

Saliva is a necessary part of the oral environment. It protects epithelium from drying and acts as a mechanical cleansing solution. Glycoproteins in saliva ensure lubrication, which minimizes the abrasive action of foods and facilitates speech. These different functions of saliva are linked to its viscosity. When saliva is absent, as in xerostomic patients, an unpleasant condition is created with potentially serious consequences.

If the rheological characteristics of natural saliva can be quantified within a physiological range, then by matching such characteristics for a substitute fluid the latter can function rheologically adequately.

In a recent study it was shown that a rigid protein layer was adsorbed at the air-liquid interface [1]. The establishment of the characterization of such a layer may be helpful in assessing potential saliva substitutes. This study deals with the determination of the yield stress and flow curve of the matured protein layer.

\section{Theory}

A substance with a so-called yield stress is characterized by a solid-like (elastic) behavior at low shear stresses, and a liquid-like (viscous) behavior at high shear stresses. The stress below which solid-like behavior is apparent is called the yield stress, symbolized by $\tau_{0}$. If we assume a flow curve as shown in Fig. $1 \mathrm{a}$, the shear stress $\tau$ can be written as

$$
\tau(\dot{\gamma})=\tau_{0}+k(\dot{\gamma}) \cdot \dot{\gamma}
$$

in which $k(\gamma)$ is the "slope viscosity" and $\gamma$ the shear rate. Assuming that $\dot{\gamma}_{0}$ is the smallest measurable shear rate, the flow curve at $\dot{\gamma}<\dot{\gamma}_{0}$ cannot be verified. In other words, it is impossible to measure $\tau_{0}$ directly and the only way to estimate $\tau_{0}$ is by extrapolation to $\dot{\gamma}=0$ (e.g., both dotted lines in Fig. 1 a may be possible in that region). The apparent viscosity $\eta_{a}$ is defined by

$$
\eta_{a}=\tau / \dot{\gamma}
$$

From Eqs. (1) and (2) it can be seen that at large shear rate $\eta_{a}$ approaches $\eta_{\infty}$ (i.e., the limiting value of $k(j)$ for large $\dot{\gamma})$. If a sample is characterized by a yield stress, then $\eta_{a}$ becomes infinite at zero shear rate. The actual curve can only be guessed (see Fig. $1 \mathrm{~b})$.

Because of the arguments given above the concept "yield stress" must be used with reserve. A detailed study is given in [2]. 

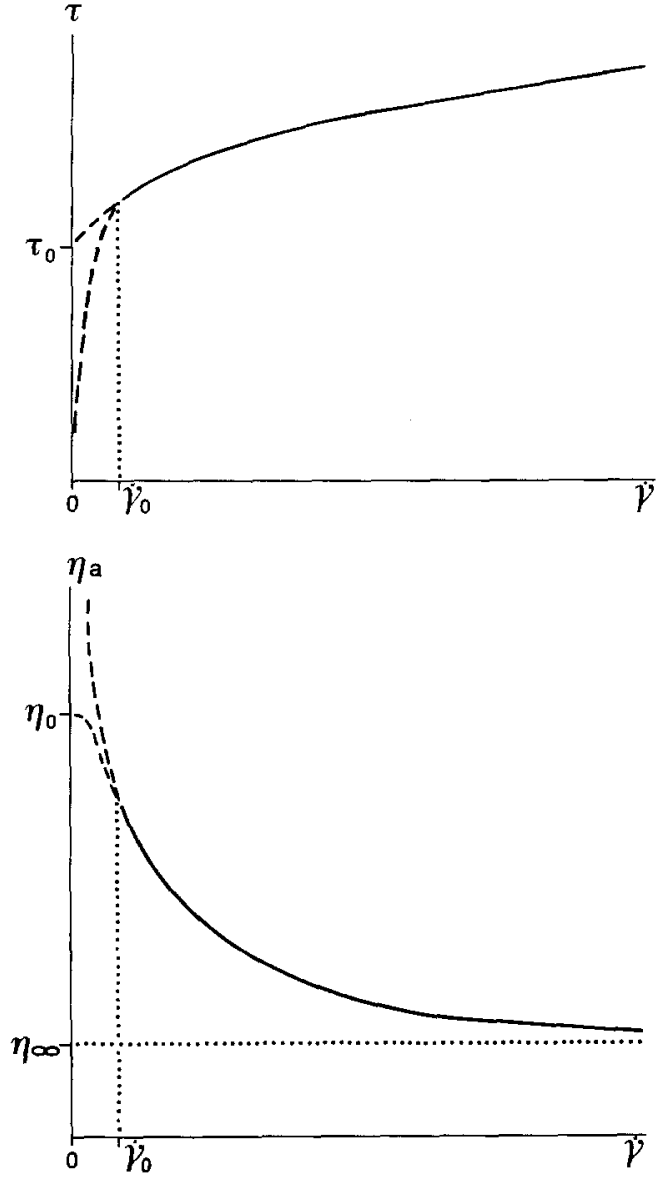

Fig. 1. (a) Flow curve of a substance with assumed yield stress $\left(\tau_{0}\right)$. The flow curve at $\gamma<\dot{\gamma}_{0}$ is unknown. (b) Corresponding $\eta_{a}(\gamma)$ curve. Below $\dot{\gamma}_{0}$ the curve is unknown. The zero-shear viscosity $\eta_{0}$ becomes infinite in case of a yield stress.

\section{Methods}

\section{Apparatus}

The apparatus used (Fig. 2) is a modification of that developed by Van Vliet et al. [3], which itself is a modification of the apparatus of Zimm and Crothers [4].

The sample is placed in a glass reservoir, which is thermostated by circulating water. To minimize evaporation during the experiment, a ridge surrounding the reservoir was filled with water, and the holder capped with an insulating glass cover. A demagnetized metal cup equipped with an eight-sector compass-card is floating on the sample. The glass cover is provided with a thin line as a reference to cup rotation. In this way angular displacements down to approximately $2^{\circ}$ could be detected $\left(\gamma_{\min }=0.04\right.$ ). The meniscus, being concave at the outer radius because of adhesion to the glass wall, should not be concave at the inner radius (i.e., at the cup), since this causes the cup to immediately stick to the glass wall. It turned out that this problem could be avoided by manufacturing the cup in such a way that it sinks exactly proportionate to its
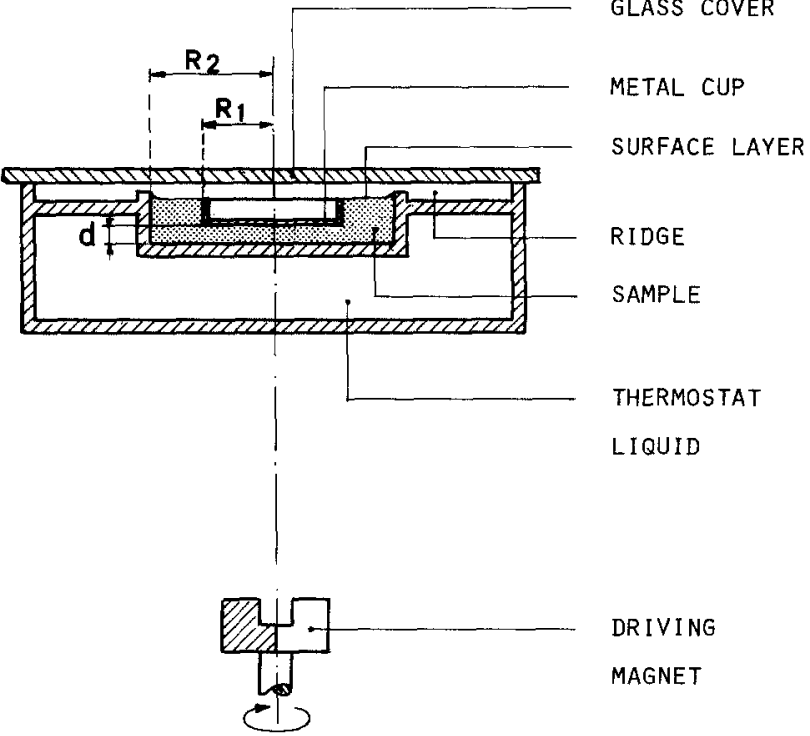

DRIVING

MAGNET

Fig. 2. Experimental design. Height of the metal cup is $4.5 \mathrm{~mm} ; \mathrm{R}_{1}$ $=12.2 \mathrm{~mm} ; R_{2}=23.2 \mathrm{~mm}$; other quantities vary.

own height into liquids with a density close to that of water $(1 \mathrm{~g} / \mathrm{ml}$ ) (see also Fig. 2).

A fast rotating (4500 rpm), permanent magnet induces a magnetic dipole in the metal cup which lags behind the dipole field of the magnet, resulting in a net torque exerted onto the cup. Its magnitude depends on the material of the cup, its dimensions, and the distance to the driving magnet. This phenomenon is caused by two effects. The main effect is the magnetic hysteresis in ferromagnetic materials, while a minor effect, but general to all metals, is caused by eddy currents which tend to counteract the rotation of the induced magnetic field in the cup. Four cups of the same dimensions made of different metals can be ranked with increasing torque under the same conditions: aluminium, brass, copper, and steel. Use of the last metal, which is the only ferromagnetic material in this sequence, resulted in about 100 times larger torque than the first three metals. In all subsequent measurements the steel cup was used. To prevent the cup from rusting it was coated with Mobil SH-01346-006. A compensation magnet above the reservoir is adjusted to minimize the influence of the Earth's magnetic field.

The distance $d$ between the cup and the bottom of the reservoir is measured using a cathetometer, the vertical displacements of which can be read with an accuracy of $0.02 \mathrm{~mm}$. The reservoir and both magnets are mounted on a small table which can be adjusted to level the bottom of the reservoir.

\section{Calibration}

The rotating driving magnet exerts a torque $M_{a}$ on the cup. If the permanent magnet rotates at a fixed speed this torque $M_{a}$ is only a function of the distance $h$ between the driving magnet and the (bottom of the) cup. This is called the "calibration function". The torque $M_{a}$ equals the counteracting torque due to the fluid and, if present, the surface layer. In a calibration experiment in which the cup floats on a Newtonian fluid (no surface layer) the torque $M_{a}$ can be calculated from 


$$
M_{a}(h)=\frac{\pi^{2} \eta R_{1}^{4}}{T_{0}(h) d}\left(1+d / d_{0}\right),
$$

where $d$ is the distance between the bottom of the cup and the bottom of the sample holder, $T_{0}$ is the period of revolution of the cup, $\eta$ is the Newtonian viscosity (Eq. (2)) of the liquid, $R_{1}$ the outer radius of the cup, and $d_{0}$ is a constant that can be determined experimentally. The first term on the righthand side of this equation represents the shear torque $M_{\text {bot }}$ onto the bottom of the cup due to the fluid underneath, while the second term represents the contribution of the counteracting torque $M_{e}$ ("edge effect") from the fluid exerted onto the cylindrical part of the disc. A detailed discussion is given in the Appendix.

\section{Surface viscosity measurement}

In case there is an adsorbed layer present at the surface the applied torque $M_{a}$, deduced at the adjusted $h$ from the calibration function, must be matched by the torques $M_{b}$ and $M_{s}$, due to the bulk liquid and the surface layer, respectively. $M_{b}$ can be calculated by the righthand side of Eq. (3) with $\eta$ the viscosity of the bulk liquid. Then $M_{s}$ can be calculated from the difference between $M_{a}$ and $M_{b}$. The viscosity $\eta_{s}$ of the surface layer can be determined as follows. The shear stress $\tau_{s}$ exerted onto the surface layer by the cup is given by

$$
\tau_{s}=\frac{M_{s}}{2 \pi R_{1}^{2} d_{s}},
$$

where $d_{s}$ is the thickness of the surface layer [5]. Assuming that the surface layer can be considered as a Newtonian liquid within the range of shear rates present in the layer, the shear rate $\psi_{s}$ at radius $R_{1}$ is given by

$$
\dot{\gamma}_{s}=\frac{4 \pi}{T_{0} \cdot\left(1-R_{1}^{2} / R_{2}^{2}\right)}
$$

where $R_{2}$ is the inner radius of the reservoir [5]. The viscosity $\eta_{s}$ of the surface layer is calculated then from the ratio of $\tau_{s}$ and $\dot{\gamma}_{s}$, giving

$$
\eta_{s}=\left(M_{a}-M_{b}\right) \cdot \frac{T_{0}\left(1 / R_{1}^{2}-1 / R_{2}^{2}\right)}{8 \pi^{2} d_{s}} .
$$

\section{Materials}

Three types of human saliva were studied: submandibular (SUB), parotid (PAR), and whole (or total) (WHL) saliva. Saliva samples $(N=22)$ from five healthy males (mean age 35 years, range $25-44$ years) were used. Saliva secretion was started at about $1 \mathrm{~h}$ after breakfast and was stimulated by a citric acid solution dripped frequently onto the tongue in the case of submandibular and parotid saliva. For the collection of whole saliva mechanical stimulation was applied by chewing on a piece of parafilm. Before starting saliva collection the mouth was rinsed with water. To perform simultaneously ellipsometric [6] measurements in which the same saliva sample was used, about $28 \mathrm{ml}$ was needed of each sample. To fill the reservoir for the flow curve measurements about
$8 \mathrm{mI}$ saliva was used. Submandibular saliva was collected using a modified Schneyer's apparatus [7]. Parotid saliva was collected using a set of modified Lashley's cups [8]. All measurements were performed at $25^{\circ} \mathrm{C}$.

\section{Experiments}

\section{Calibration and accuracies}

For the calibration of the viscometer two Newtonian liquids, dibutylphthalate (DBP) and diethylhexylphthalate (DEHP), were used with approximately the same density as water. The viscosities of DBP and DEHP at $25^{\circ} \mathrm{C}$ are $16.2 \mathrm{mPa} \cdot \mathrm{s}$ and $58.0 \mathrm{mPa} \cdot \mathrm{s}$, respectively [9].

For the calibration with DBP and DEHP the distance $h$ was varied between 20 and $45 \mathrm{~mm}$. The deduced $M_{a}(h)$ (see Appendix) curve is shown in Fig. 3: the calibration function. The following empirical relation for $M_{a}(h)$ holds

$$
M_{a}(h)=C\left(h / h_{1}\right)^{a}
$$

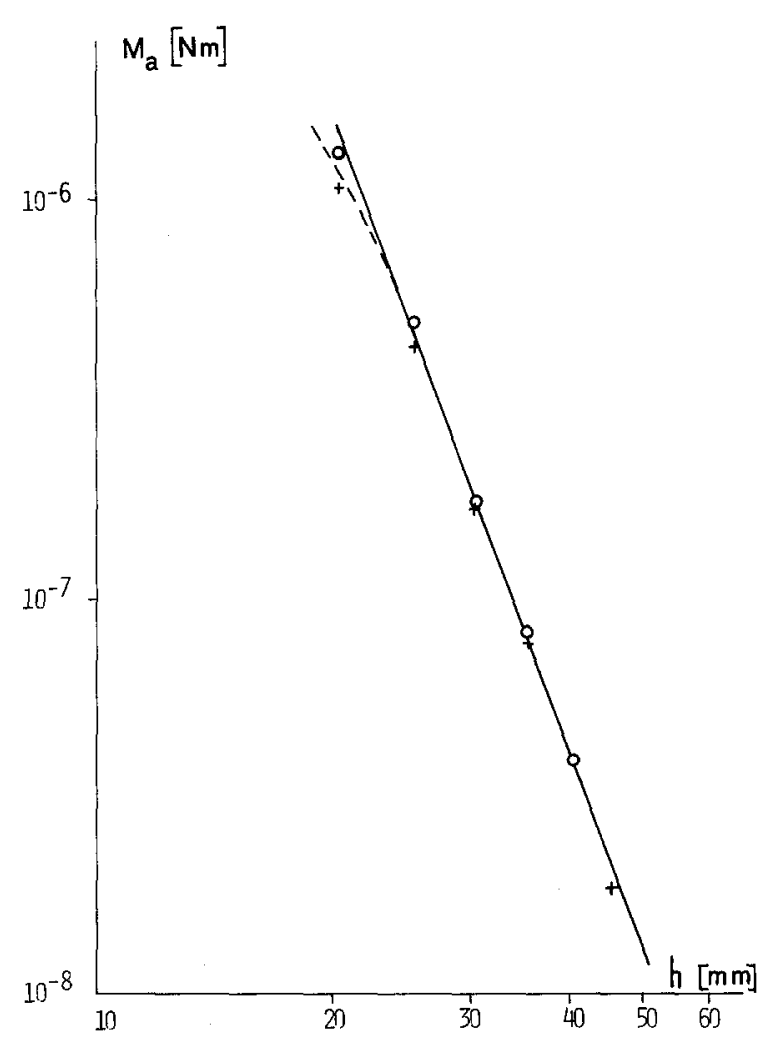

Fig. 3. Calibration curve: the total applied torque $M_{a}$ as a function of the distance $h$. 
In this equation, $h_{1}$ represents an arbitrary value of $h$, for reference only; $C$ and $\alpha$ are constants, where $C$ depends on the choice of $h_{1}$, whereas $\alpha$ is calculated from the plot of $\log \left(M_{a}\right)$ vs $\log (h)$ :

$$
\alpha=-5.6 \pm 0.2
$$

A torque $M_{a}$ ranging from $10^{-8}$ to $10^{-6} \mathrm{Nm}$ could be applied. Larger torque values could not be applied in the chosen configuration (magnetic strength of the driving magnet, dimensions and material of the steel cup). Using $\Delta h \sim 0.02 \mathrm{~mm}$, the accuracy with which $M_{a}(h)$ can be adjusted is $\Delta M_{a} / M_{a} \leq 0.08$. The determination (see Appendix) of $d_{0}$ from the measurements given in the is:

$$
d_{0}=0.75 \pm 0.07 \mathrm{~mm}
$$

It appeared that Eq. (3) was valid for $d$ values up to about $2 \mathrm{~mm}$ (see Appendix and Fig. 5).

For the determination of the viscosity of the surface layer the applied $M_{a}(h)$ can be found from Fig. 3 . The next step is to calculate the torque $M_{b}$ with aid of Eq. (3). Finally, the viscosity of the surface layer $\eta_{s}$ is found from Eq. (6). Propagation error analysis teaches that $M_{b}$ for a distance $d=1.5 \mathrm{~mm}$ as used in the experiments can be calculated with a relative error

$$
\Delta M_{b} / M_{b}=0.1+\frac{\Delta \eta}{\eta}
$$

The viscosity of the surface layer can be found with a relative accuracy

$$
\frac{\Delta \eta_{s}}{\eta_{s}} \approx 0.2
$$

where typical errors in $\eta, T_{0}, R_{1}, R_{2}$, and $d_{s}$ are used.

Three additional remarks should be made. In the foregoing, Newtonian character of the liquids was assumed. The effect of non-Newtonian character is addressed in the discussion.

The bulk viscosity $\eta_{b}$ is not known a priori, but can be determined in a separate experiment (see discussion).

Further, one must keep in mind that the biological variation between the samples caused a spread between points of various flow curves at the same rate of shear which is much larger than the calculated errors in the points of one curve.

\section{Characterization of saliva samples}

In a parallel ellipsometric experiment [7] the thickness $d_{s}$ and index of refraction $n_{s}$ of the adsorbed layer of the same samples as investigated here were determined. In addition, the amount adsorbed material per unit area $\Gamma$ and the surface concentration $c_{s}$ $=\Gamma / d_{\mathrm{s}}$ could also be given [6]. A survey of these parameters is given in Table 1.

Table 1. Classification scheme based on $c_{s}$ values $\left(T=25^{\circ} \mathrm{C}\right)$

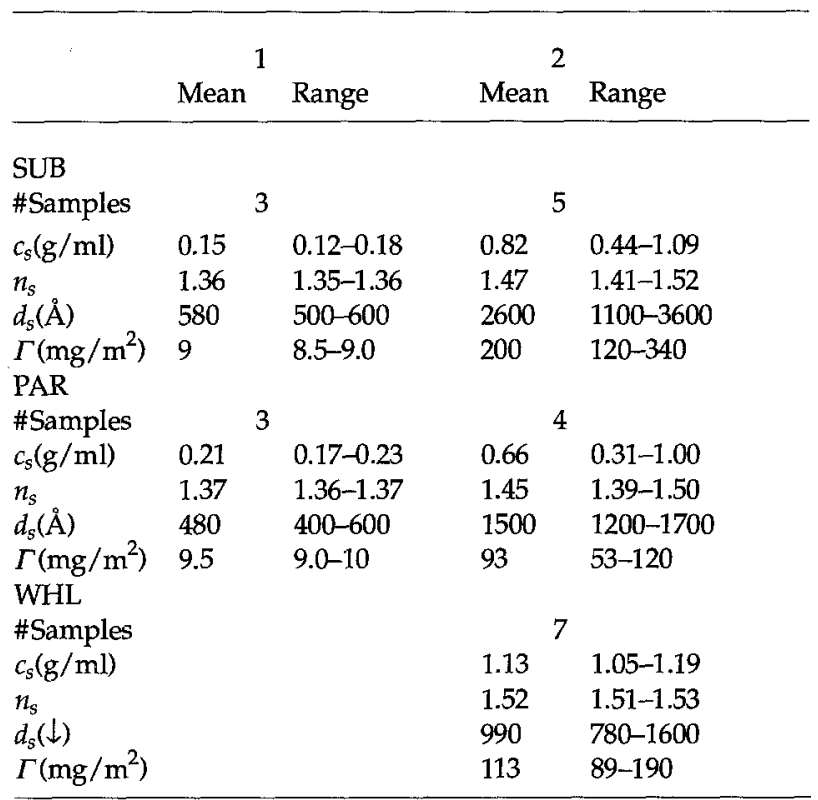

Abbreviations: $\mathrm{SUB}=$ submandibular; $\mathrm{PAR}=$ parotid; $\mathrm{WHL}=$ whole; \# = number of samples. Note: $\Gamma$ is calculated using $n_{\text {solv }}=$ 1.3327 and $d n / d c=0.17 \mathrm{ml} / \mathrm{g}$. Other quantities are discussed in [7].

Further characterization of the samples is given with aid of the bulk properties of the fluid underneath the surface layer as index of refraction $n_{b}$, density $\rho$, acidity $\mathrm{pH}$, and electrical conductivity $\Lambda$ (see Table 2).

\section{Saliva flow-curve measurements}

Within $30 \mathrm{~min}$ after secretion had finished the reservoir was filled with $8 \mathrm{ml}$ of the saliva sample ( $d$ $=1.5 \mathrm{~mm}$ ). The metal cup was carefully placed into the sample using a pair of plastic tweezers to avoid magnetization of the cup. The driving magnet was placed in its lowest position. 
Table 2. Bulk properties classified corresponding to the classification of Table $1\left(\mathrm{~T}=25^{\circ} \mathrm{C}\right)$

\begin{tabular}{|c|c|c|c|c|}
\hline & \multicolumn{2}{|c|}{1} & \multicolumn{2}{|c|}{2} \\
\hline & Mean & Range & Mean & Range \\
\hline \multicolumn{5}{|l|}{ SUB } \\
\hline \#Samples & \multicolumn{2}{|c|}{3} & \multicolumn{2}{|c|}{5} \\
\hline $\begin{array}{l}\text { Secretion rate } \\
(\mathrm{ml} / \mathrm{min})\end{array}$ & 0.9 & $0.73-1.1$ & 0.85 & $0.63-1.1$ \\
\hline$n_{b}$ & 1.3333 & $1.3330-1.3335$ & 1.3332 & $1.3330-1.3335$ \\
\hline$\rho\left(\mathrm{kg} / \mathrm{m}^{3}\right)$ & 999.3 & $998.8-999.5$ & 999.3 & $998.5-1000.1$ \\
\hline $\mathrm{pH}$ & 6.9 & $6.8-7.1$ & 7.6 & $7.3-7.9$ \\
\hline$\Lambda\left(10^{-3} \Omega^{-1} \mathrm{~cm}^{-1}\right)$ & 3.2 & $2.4-3.6$ & 3.8 & $2.4-6.0$ \\
\hline PAR & & & & \\
\hline \#Samples & \multicolumn{2}{|c|}{3} & \multicolumn{2}{|c|}{4} \\
\hline $\begin{array}{l}\text { Secretion rate } \\
(\mathrm{ml} / \mathrm{min})\end{array}$ & 0.35 & $0.34-0.35$ & 1.5 & $1.1-1.9$ \\
\hline$n_{b}$ & 1.3336 & $1.3333-1.3340$ & 1.3338 & $1.3338-1.3340$ \\
\hline$\rho\left(\mathrm{kg} / \mathrm{m}^{3}\right.$ & 1000.2 & $999.4-1000.9$ & 1000.7 & $998.9-1001.9$ \\
\hline $\mathrm{pH}$ & 6.4 & $5.1-7.4$ & 8.2 & $8.1-8.4$ \\
\hline$\Lambda\left(10^{-3} \Omega^{-1} \mathrm{~cm}^{-1}\right)$ & 3.5 & $3.0-3.8$ & 5.9 & $5.4-7.0$ \\
\hline WHL & & & & \\
\hline \#Samples & & & \multicolumn{2}{|c|}{7} \\
\hline Secretion rate & & & & \\
\hline$(\mathrm{ml} / \mathrm{min})$ & & & 0.74 & $0.28-0.9$ \\
\hline$n_{b}$ & & & 1.3333 & $1.3332-1.3335$ \\
\hline$\rho\left(\mathrm{kg} / \mathrm{m}^{3}\right)$ & & & 999.3 & $999.0-1000.0$ \\
\hline $\mathrm{pH}$ & & & 7.5 & $7.4-7.7$ \\
\hline$\Lambda\left(10^{-3} \Omega^{-1} \mathrm{~cm}^{-1}\right)$ & & & 3.3 & $2.9-4.0$ \\
\hline
\end{tabular}

Note: The secretion rate is the rate at which the saliva has been produced.

Measurements were started about $3 \mathrm{~h}$ after filling of the reservoir to insure that the thickness of the surface layer was constant [7]. In the starting position of the driving magnet the driving torque $M_{a}$ was about $10^{-8} \mathrm{Nm}$. To sufficiently accurately obtain the angular displacement time intervals up to about 15 min were used at low shear rates. At higher shear rates the time intervals were about $5 \mathrm{~min}$. The shear stress was calculated from Eq. (4) using the layer thickness determined by ellipsometry [7]. The shear rate was calculated from Eq. (5). The applied torque was increased until the shear rate reached large values $\left(>10^{-2} \mathrm{~s}^{-1}\right)$. This point was reached within $4-7$ $\mathrm{h}$ after filling.

\section{Discussion}

As indicated in the theory section, the determination of the yield point is an unsolvable extrapolation problem. Here samples showing a sudden breakdown resulting in a zero $\tau(j)$ slope over a large $\gamma$ range up to $0.1 \mathrm{~s}^{-1}$ are supposed to have a yield point.
In 14 out of 22 samples no such yield point could be observed and, instead, a gradual flow occurred.

The results are listed in Table 3 . From this it can be seen that most layers on whole saliva present a yield stress, while no yield stress was observed for samples of parotid saliva. The lowest shear stress $\left(\tau_{\min }\right)$ at which cup rotation could be observed at the smallest measurable shear rate is, on the average, significantly higher in the samples showing a yield stress than in the samples without a yield stress. Particularly, surface layers on parotid saliva show small $\tau_{\min }$ values.

Table 3. Classification according to yield point occurrence. $(\mathrm{T}=$ $25^{\circ} \mathrm{C}$.)

\begin{tabular}{|c|c|c|c|c|c|c|c|}
\hline & & \multicolumn{2}{|c|}{ SUB $(N=8)$} & \multicolumn{2}{|c|}{$\operatorname{PAR}(\mathbb{N}=7)$} & \multicolumn{2}{|c|}{ WHL $(N=7)$} \\
\hline & & Mean & Range & Mean & Range & Mean & Range \\
\hline \multirow{3}{*}{$\begin{array}{l}\text { Yield } \\
\text { point }\end{array}$} & $\mathrm{N}$ & \multicolumn{2}{|c|}{3} & \multicolumn{2}{|c|}{0} & \multicolumn{2}{|c|}{5} \\
\hline & $\tau_{\mathrm{y}}[\mathrm{kPa}]$ & 8.9 & $1.7-13$ & - & - & 22 & $7.4-35$ \\
\hline & $\mathrm{N}$ & \multicolumn{2}{|c|}{5} & \multicolumn{2}{|c|}{7} & \multicolumn{2}{|c|}{2} \\
\hline $\begin{array}{l}\text { Gradual } \\
\text { flow }\end{array}$ & $\tau_{\min }[\mathrm{kPa}]$ & 5.1 & $1.3-11$ & 0.39 & $0.06-1.2$ & 6.1 & $2.9-9.3$ \\
\hline
\end{tabular}

Abbreviations: $N=$ number of samples included; $S U B=$ submandibular; $P A R=$ parotid; $W H L=$ whole saliva.

For samples with a surface layer not showing an apparent yield point the plot of $\log \left(\eta_{s}\right)$ vs $\log \left(\dot{\gamma}_{s}\right)$ shows a largely linear dependence (Fig. 4). This linear behavior reveals a power-law dependence:

$$
\eta_{s}=c \dot{\gamma}_{s}^{\beta}
$$

in which $c$ is a constant that appears to be different for each sample. The related equation for the shear stress, using Eq. (2) is

$$
\tau_{s}=c \gamma_{s}^{\beta+1} \text {. }
$$

For all samples the slopes $\beta$ of the plots are:

$$
\beta=-0.93 \pm 0.05 \text {, }
$$

which has been corrected for a small contribution of the bulk liquid (see the following).

The zero-shear viscosity $\eta_{s 0}$ may be only roughly estimated by extrapolation to lower $\dot{\gamma}_{s}$. Although there is no significant difference in $\beta$ with respect to the type of saliva, the zero-shear viscosity seems to differ appreciably (Table 4). 


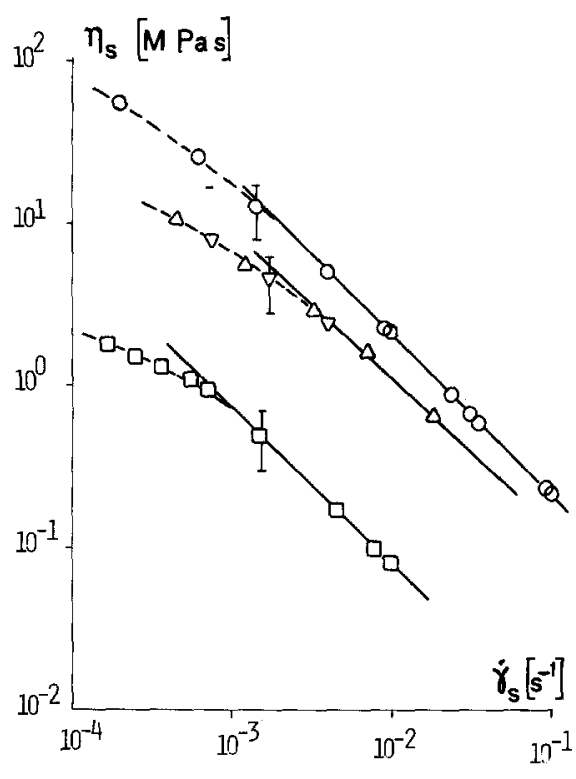

Fig. 4. Three typical curves of the apparent viscosity as a function of the shear rate. Note the linear slope at higher shear rates. ( $\square$ : parotid saliva; $0, \nabla$ : submandibular saliva).

Table 4. Approximate values of the zero-shear viscosity of saliva from the experiments that showed gradual flow. $\left(T=25^{\circ} \mathrm{C}\right.$.)

\begin{tabular}{lll}
\hline & Number of samples included & $\eta_{\text {so }}[\mathrm{mPa} \cdot \mathrm{s}]$ \\
\hline SUB & 5 & $10-100$ \\
PAR & 7 & $1-10$ \\
WHL & 2 & $\sim 100$ \\
\hline
\end{tabular}

In using Eqs. (5) and (6) to determine the flow curves the surface layer is considered to be Newtonian. Obviously, in the power-law region this is not the case. Recalculation using the formulas for a power-law fluid [5] resulted in flow curves which appeared to be merely shifted along the $\dot{\gamma}_{s}$ axis.

To estimate the bulk contribution $M_{b}$ in Eq. (6) it is necessary to estimate the viscosity of the bulk liquid as a function of shear rate. Therefore, steadystate measurements were performed in which the influence of the surface layer could be eliminated (a detailed description of the method used is given by Waterman et al. [10]). It turned out that $M_{b}$ could be safely neglected. It did not change the rough estimates of the zero-shear viscosity, and the power-law exponent $\beta$ was affected less than $3 \%$. The value of $\beta$ is in good agreement with the results from steadystate measurements of various polymer solutions, showing a decrease in the power-law exponent with concentration, the exponent tending to approach a value close to -1 for systems with $c_{s}[\eta] \geq 50[11]$ as is the case here ([ $\eta]$ is the intrinsic viscosity).

If one assumes a yield stress $\tau_{s o}$ added to Eq. (9), then

$$
\tau_{s}=\tau_{s 0}+c \dot{\gamma}_{s}^{\beta+1}
$$

and this would give rise to an extra increase in $\tau / \gamma$ at decreasing $\dot{\gamma}$. Because the opposite is observed, that is, $\eta_{s}$ tends to a constant value at low shear rates, the experiments do not support the existence of a non-zero $\tau_{0}$ value.

Striking is the difference between parotid saliva compared to submandibular and whole saliva. No apparent yield stress and lower estimates of zero shear viscosities occur for parotid saliva, whereas whole and submandibular saliva sometimes showed yield stresses and high estimates of the zero shear viscosity.

Influence of acidity is improbable (Table 2). Saliva contains proteins, electrolytes, and small organic molecules [12]. For the adsorption phenomenon and the yield or flow behavior of the adsorption layer the proteins are probably responsible. The main difference between PAR and SUB/WHL is found in the high molecular components: mainly mucins for submandibular and whole saliva, and globular proteins for parotid saliva (Table 5). Mucins and globular proteins differ at least in two respects that affect the rheological behavior of saliva. Mucins consist of a backbone of amino acids with oligosaccharides as side chains. The side chains contain sialic acid end groups which are negatively charged for $\mathrm{pH}>6$. Consequently, the mucin molecule, though flexible, is more or less stretched. Its molecular weight is > $5.10^{5}$ Dalton; this is quite different from globular proteins. Parts of the latter are folded into domains, thus the molecule is rather compact. Its molecular weight is typically on the order of $10^{5}$ Dalton.

The rheological effect of both factors in absence of a pH effect is consistent with the observed (absence of yield and flow behavior of the adsorbed layer: stretched molecules of high molecular weight at high concentration more easily show yield behavior and high viscosity under flow conditions than more compact molecules of lower molecular weight. 
Table 5. Quantitatively most important protein components in unstimulated saliva [12]

\begin{tabular}{lccc}
\hline Protein (mg/100ml) & PAR & SUB & WHL \\
\hline Enzymes & & & \\
a-Amylase & $40-80$ & 30 & 40 \\
Lysozyme & 2 & 1.5 & 2 \\
Kallikrein & & & 2 \\
Lactoperoxidase & & & 1 \\
Proline-rich proteins (PRP) & 50 & 50 & 50 \\
Acidic PRP & 30 & 30 & 30 \\
Basic PRP & 30 & - & 20 \\
Basic glycoprotein & & & \\
High-molecular components & - & 70 & 50 \\
Mucins & 10 & & 19 \\
Immunoglobulins & & & 4.2 \\
IgA & & & 0.6 \\
IgG & & \\
IgM & & & 1 \\
Minor components & & - \\
Lactoferrin & 3 & 2 \\
Gustin & & \\
\hline
\end{tabular}

PAR = parotid saliva, SUB $=$ submandibular $s ., W H L=$ whole $s$.

Considering the development of a substitute fluid for saliva that rheologically mimics saliva, the composition of whole saliva and the results of the present investigation suggest the use of mucins in a substitute.

\section{Appendix}

At equilibrium the applied torque $M_{a}$ must be equalled by the total counteracting torque from the Newtonian liquid (no surface layer present). The latter can be divided into the plate-plate shear torque $M_{\text {bot }}$ onto the bottom of the cup and the torque $M_{e}$ to account for other contributions ("edge effect"):

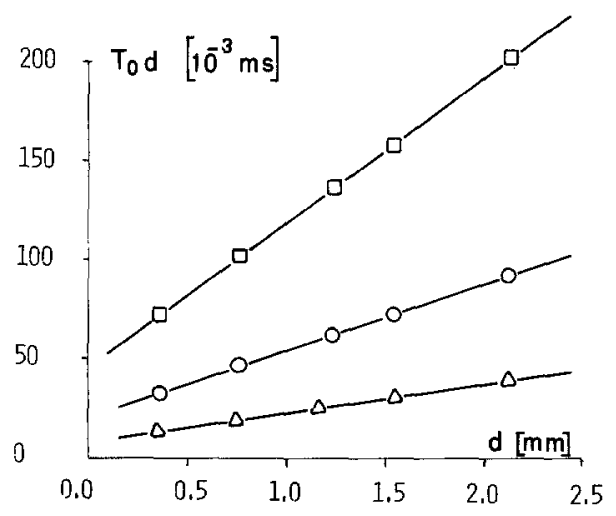

Fig. 5. Three typical curves of $T_{0} \cdot d$ vs $d$ from measurements with DBP at constant $h_{0} .\left(\square: h_{0}=35.5 \mathrm{~mm} ; O: h_{0}=30.5 \mathrm{~mm} ; \triangle: h_{0}=25.5\right.$ $\mathrm{mm}$ ).

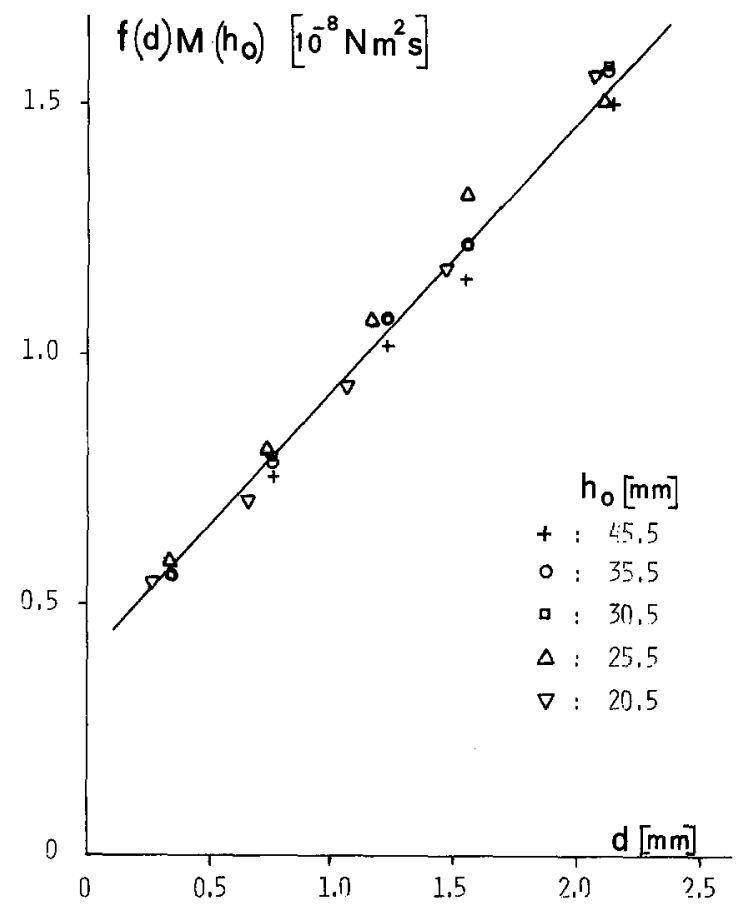

Fig. 6. Master calibration line for $T_{0} d M_{a}\left(h_{0}\right)$ vs $d$ to demonstrate the consistency of the method.

$$
M_{a}=M_{\text {bot }}+M_{e}
$$

The torque $M_{\text {bot }}$ is given by

$$
M_{\text {bot }}\left(T_{0}, d\right)=\frac{\pi^{2} \eta R_{1}^{4}}{T_{0} d}
$$

where $\eta$ is the viscosity of the liquid, $R_{1}$ is the cup radius, $T_{0}$ the period of revolution of the cup, and $d$ is the distance between the cup and the bottom of the reservoir [5]. For convenience the quantity $r$ is defined by

$$
r=M_{e} / M_{\text {bot }}
$$

Obviously, at decreasing $d$ the counteraction of the liquid will increase and, thus, the time $T_{0}$ must increase when the applied torque is kept constant. $M_{e}$ does not depend on $d$ for small values of $d$, but on $T_{0}$ only. If $M_{e}$ decreases, $M_{\text {bot }}$ will increase to match Eq. (A1). Thus the ratio $r$ is a function of $d$ and becomes zero at $d=0$. Defining the function $f(d)=T_{0} d$, it can be deduced, from Eq. (A1) through (A3) that

$$
f(d) \cong \frac{\pi^{2} \eta R_{1}^{4}}{M_{a}(h)} \cdot(1+k d)
$$

where the approximation $r \cong k \cdot d$ is used, valid for small values of $d$. Subsequently, one can expand $M_{a}(h)$ in a Taylor series around $h_{0}$ with $h=h_{0}+d$ and substitute the first two terms in (A4), then 


$$
f(d) \cong \frac{\pi^{2} \eta R_{1}^{4}}{M_{a}\left(h_{0}\right)}\left(1+\left(k-\frac{1}{M_{a}\left(h_{0}\right)} \cdot \frac{d M_{a}}{d h}\left(h_{0}\right)\right) d\right) .
$$

From a plot of $T_{0} d(=f(d))$ vs $d$ (see Fig. 5) at $d=0, M_{a}\left(h_{0}\right)$ can be deduced.

The plot of $M_{a}$ vs $h_{0}$ turns out to be of the form $M_{a}=A h_{0}{ }^{\alpha}$ with $A$ and $\alpha$ constants $(\alpha=-5.6 \pm 0.2)$. No significant loss of accuracy occurred when $h_{0}$ was replaced by $h$ (Fig. 3). From the slope of $f$ (d) vs $d$ follows $k-\frac{1}{M_{a}\left(h_{0}\right)} \frac{d M_{a}}{d h}\left(h_{0}\right)$, and with Fig. $3 k=1 / d_{0}$ can be found. The consistency of this approach is demonstrated in Fig. 6 where $f(d) M_{a}(h)$ vs $d$ has been plotted.

\section{References}

1. Waterman HA, 's-Gravenmade EJ, van der Wallen Mijnlieff PF (1985) J Dental Res 64:716

2. Barnes HA, Walters K (1985) Rheol Acta $24: 323$

3. Van Vliet T, de Groot-Mostert AEA, Prins A (1981) J Phys E Sci Instrum 14:745

4. Zimm BH, Crothers DM (1962) Proc Nat Acad Sci US 48:905

5. Whorlow RW (1980) Rheological Techniques, Ellis Horwood Ltd, Wiley \& Sons, Chichester: Ch 3
6. Holterman HJ, 's-Gravenmade EJ, Waterman HA, Mellema J, Blom C (1989) J Coll Interf Sci 128:523

7. Schneyer LH (1955) J Dental Res 34:257

8. Lashley KS (1916) J Exp Psychol 1:461

9. Blom C, Mellema J (1984) Rheol Acta 23:98

10. Waterman HA, Blom C, Holterman HI, 's-Gravenmade EI, Mellema J (1988) Arch Oral Biol 33:589

11. Graessley WW (1974) Fortschr Hochpolym-Forsch $16: 1$

12. van Nieuw Amerongen A (1988) Saliva and salivary glands. Its importance in oral health (in Dutch), Samson Stafleu, Alphen aan de Rijn, Brussel

Received accepted

Authors' address:

\section{H. J. Holterman}

Rheology Group

Faculty of Applied Physics

University of Twente

Enschede, The Netherlands 